\title{
HSCT FOR PEDIATRIC DISEASES
}

\begin{abstract}
Adriana Seber $17,18,16,27$, Alessandra Gomes ${ }^{25,22}$, Ana Karine Vieira ${ }^{7}$, Ana Luiza Melo Rodrigues ${ }^{11}$, Ana Maria Marinho da Silva ${ }^{2}$, Antonella Zanette ${ }^{11}$, Antonio Vaz de Macedo ${ }^{6,13}$, Carla Nolasco Monteiro Breviglieri ${ }^{17,20,25}$, Carla Zanchetta ${ }^{17,19}$, Cilmara Kuwahara ${ }^{15}$, Cinthya Rocha Carvalho ${ }^{23}$, Fernanda Lima Lelis $^{21}$, Gabriele Zamperlini Netto ${ }^{12,25}$, Gustavo Zamperlini ${ }^{17,19}$, Julia Lopes Garcia'12,25, Juliana Folloni Fernandes ${ }^{12,25}$, Karoline Helena Silva da Silva ${ }^{24}$, Laila Rigolin Fortunato ${ }^{5}$, Lauro José Gregianin ${ }^{10}$, Liane Esteves Daudt ${ }^{32}$, Luciana Domingues ${ }^{17,20}$, Luiz Guilherme Darrigo Jr ${ }^{31}$, Maria Gabriela Matos ${ }^{17}$, Maria Lucia Lee ${ }^{3}$, Mariana Bohns Michalowski ${ }^{32}$, Maura Ikoma', Monica Cypriano ${ }^{24}$, Natália Maria Tavares Ferreira Borges ${ }^{21}$, Neysimelia Costa Villela ${ }^{8}$, Paola Soriano ${ }^{17,20}$, Patrícia Shimoda Ikeuti ${ }^{8}$, Paulo Henrique dos Santos Klinger ${ }^{24}$,Raul Ribeiro ${ }^{30}$, Renata Fittipaldi Guimaraes ${ }^{24}$, Rita Barbosa Tavares ${ }^{26}$, Roseane Gouveia ${ }^{17,19}$, Samantha Nichele" ${ }^{9}$, Simone de Castro Resende Franco ${ }^{4}$, Valeria Cortez

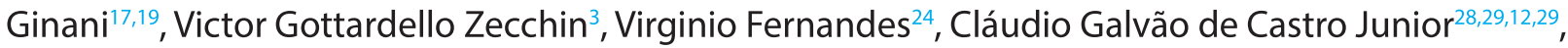
Carmem Bonfim ${ }^{15}$
\end{abstract}

\begin{abstract}
1 Hospital Amaral Carvalho - Jaú - SP - 2 Hospital Aristides Maltez - Salvador - BA- 3 Hospital Beneficência Portuguesa - São Paulo - SP - 4 Hospital da Criança de Brasília - Brasília - DF - 5 Hospital da Criança e Maternidade - São José do Rio Preto - SP 6 Hospital da Polícia Militar - Belo Horizonte - MG - 7 Hospital das Clínicas - Universidade Federal de Minas Gerais - Belo Horizonte - MG - 8 Hospital de Amor Infanto Juvenil de Barretos - Barretos - SP - 9 Hospital de Clínicas - Universidade Federal do Paraná - Curitiba - PR - 10 Hospital de Clínicas de Porto Alegre - Porto Alegre - RS - 11 Hospital Erastinho - Complexo Erasto Gaertner - Curitiba - PR - 12 Hospital Israelita Albert Einstein - São Paulo - SP - 13 Hospital Luxemburgo - Instituto Mário Penna - Belo Horizonte - MG - 14 Hospital Martagão Gesteira - Salvador - BA - 15 Hospital Pequeno Príncipe - Curitiba - PR 16 Hospital Sabará - São Paulo - SP - 17 Hospital Samaritano Higienópolis - São Paulo - SP - 18 Hospital São Camilo - São Paulo - SP - 19 Hospital São Camilo Pompéia - São Paulo - SP - 20 Hospital São Camilo Santana - São Paulo - SP 21 Hospital São Rafael - Salvador - BA - 22 Hospital Sírio Libanês - 23 Instituto de Medicina Integral Professor Fernando Figueira (IMIP) - Recife - PE - 24 Instituto de Oncologia Pediátrica - GRAACC - UNIFESP - São Paulo - SP - 25 Instituto do Tratamento do Câncer Infantil ITACI - Hospital das Clínicas - Universidade de São Paulo - São Paulo - SP 26 Instituto Nacional do Cancer - Rio de Janeiro - RJ - 27 Pediatric HCT Committee - Sociedade Brasileira de Oncologia Pediátrica (SOBOPE) and Sociedade Brasileira de Transplante de Medula Óssea (SBTMO) - 28 Santa Casa de Misericórdia de Porto Alegre - Porto Alegre - RS - 29 Sociedade Brasileira de Oncologia Pediátrica - São Paulo - SP - 30 St. Jude Children's Research Hospital - Memphis - TN - 31 Universidade de São Paulo - Ribeirão Preto - SP - 32 Universidade Federal do Rio Grande do Sul - Porto Alegre - RS
\end{abstract}

The Brazilian Society of Bone Marrow Transplantation (SBTMO) started in 2009 ${ }^{[1]}$ its Consensus Meetings to discuss the indications for Hematopoietic Stem Cell Transplantation (HCT), and the resulting consensus guidelines have ever since remained freely available at the SBTMO website (www.sbtmo.org.br/consenso). ${ }^{[2]}$ Now, in its 5 th edition, the Consensus material will, for the first time, be published in the Journal of Bone Marrow Transplantation and Cellular Therapy (JBMTCT - www.jbmtct.com.br) recently launched by SBTMO, the first scientific journal in Latin America focused on $\mathrm{HCT}^{[3]}$ The recommendations will remain in Portuguese at the SBTMO website, and in English at the Journal website.

All prior meetings have been presential except for this one, on October 26,2020 , initially planned to be hosted in the city of São Paulo, immediately before the XXIV
SBTMO Annual Meeting, and modified to a digital platform (www.congressosbtmo.org.br) due to the SARSCov2 pandemic, as has been the case with so many other national and international meetings in the field.

Although a significant impact of COVID-19 on the activity of solid organ transplantation has been witnessed in the country over the past few months, when comparing the first half of 2019 to that of 2020 , an overall reduction of only $10 \%$ in allogeneic HCT was observed, ${ }^{[4]}$ which highlights the critical relevance of this procedure and the boundless commitment of both donors and healthcare professionals to the treatment of an array of life-threatening diseases that may be effectively treated with a timely transplant. Hence, the annual number of transplants has been growing continuously worldwide, ${ }^{[5]}$ as well as in our country. ${ }^{[4]}$ 
The addition of post-transplant cyclophosphamide to haploidentical transplantation for graft-versus-host disease (GVHD) prophylaxis, initially developed within experimental platforms ${ }^{[6]}$ has been used in clinical trials since $2002^{[7]}$ with an astounding impact on donor selection and transplantation practices worldwide. As a result, hundreds of publications on haploidentical HCT have shown very similar outcomes to those obtained with unrelated $\mathrm{HCT}^{[8,9]}$ To date, the only randomized-controlled trial available comparing double unrelated cord blood with haploidentical marrow transplantation (BMT-CTN 1101) showed a lower transplant-related mortality and better overall survival with the use of haploidentical donors. ${ }^{[10]}$

Allogeneic HCT, traditionally a therapeutic option restricted to patients with an HLA-compatible donor, is now available to virtually all who need it, since parents and children invariably share a common haplotype, in addition to half of the siblings. The worldwide and Brazilian experience acquired during the past several years with the various types of donors and grafts has enabled us to change our rationale for recommending $\mathrm{HCT}$.

Many current consensuses for HCT indications no longer differentiate between indications for transplants using grafts from HLA-identical related and haploidentical donors, adult unrelated donors, and umbilical cord blood units. ${ }^{[11]}$ Nonetheless, each transplant strategy has its own particularities, risks and benefits. When performing any HCT with HLA incompatibility, it is essential to look for donor-specific antibodies (DSA) and to have strategies to desensitize the patient if antibodies against the donor are found.

Patients with an indication for allogeneic HCT should be transplanted with the best available donor as soon as the procedure is indicated, before disease progression or deterioration of the patient's clinical status. These are the most important prognostic factors for treatment outcome.

Pediatric diseases requiring HCT have a much lower prevalence in the population than diseases affecting adults. The discovery of a myriad of specific genetic abnormalities has changed our understanding of many malignant and non-malignant pediatric diseases. When all these specificities are combined to the various types of donor and transplant strategies now available, we understand that the classical model used to define HCT indications in adults, with meta-analyses and randomized trials, are not applicable in pediatrics. In addition, many pediatric diseases do not have therapeutic alternatives with curative potential that can be compared to HCT results. Thus, in this 2020 Consensus, we chose to follow international guidelines ${ }^{[11]}$ and make recommendations for indications of autologous and allogeneic HCT, regardless of the type of graft or donor.

Members of the Pediatric Working Group participated in several meetings to discuss the specific consensuses on bone marrow failure syndromes, hemoglobinopathies, autoimmune diseases, and sinusoidal obstruction syndrome (Supplementary Table 1). These topics were not included in this document.

Six groups were formed to review the indications for non-malignant diseases and fifteen groups for the review of malignant diseases (Supplementary Table 2). All reviews were discussed during the group's weekly meetings on the www.Cure4Kids.org website, kindly offered by St. Jude Children's Research Hospital.

The main changes in relation to the previous consensus are presented in this article and were orally presented at the SBTMO - Consensus Plenary Session on October 26, 2020 for comments. A supplement of the JBMTCT will follow, discussing all Pediatric HCT indications in depth. The indications are summarized in Tables 1 to 3 and the recommendations of essential medications used to perform allogeneic HCT are listed in Table 4.

For each disease, we have defined whether autologous and/or allogeneic HCT are recommended or not and have added a few important notes on the implications regarding specific indications and the approach to performing the transplants. In the tables, the letter Y means Yes, HCT is indicated", and N means "No, it is not indicated".

Of note, we have only included the most common pediatric indications in this guideline. Diseases that have not been previously discussed as treated with $\mathrm{HCT}$ are not included in the tables, which does not mean that HCT may not be performed as an exemption or under compassionate use, as far as it is based on a strong rationale, or on documented previous $\mathrm{HCT}$ successes. A rare indication does not imply that a transplant is experimental; it means that that there are not enough patients under that indication to perform a formal clinical trial. Therefore, sound clinical judgment is advised at all times when faced with the challenge of an indication for transplant.

We thank all of those who have dedicated their time and effort toward updating these guidelines and hope that this 2020 Consensus succeeds in providing solid evidence-based guidance to all healthcare workers involved in the continuous care of HCT patients in Brazil and developing countries alike. 
TABLE 1- Indications for Allogeneic Hematopoietic Stem Cell Transplantation for Non-Malignant Diseases in Pediatrics

\begin{tabular}{|c|c|c|c|}
\hline & Observations/indications & Allo & Auto \\
\hline \multicolumn{4}{|l|}{ Inherited inborn errors of metabolism } \\
\hline Osteopetrosis & $\begin{array}{l}\text { Urgent HCT due to the risk of blindness and hearing loss, } \\
\text { except in the presence of neurodegeneration (OSTM1 } \\
\text { mutation) and with RANKL mutations }\end{array}$ & Y & $\mathrm{N}$ \\
\hline $\begin{array}{l}\text { Mucopolysaccharidosis type I- MPS-IH, } \\
\text { Hurler syndrome }\end{array}$ & & Y & $\mathrm{N}$ \\
\hline $\begin{array}{l}\text { Mucopolysaccharidosis type II, } \\
\text { Hunter syndrome }\end{array}$ & & Y & $\mathrm{N}$ \\
\hline $\begin{array}{c}\text { Mucopolysaccharidosis type VI, Maroteaux-Lamy } \\
\text { Syndrome }\end{array}$ & ONLY if unresponsive to enzyme replacement therapy & Y & $\mathrm{N}$ \\
\hline X-linked adrenoleukodystrophy & ONLY progressive cerebral form, early stage & Y & $\mathrm{N}$ \\
\hline Leukodystrophy of globoid cells - Krabbe disease & $\begin{array}{c}\text { Warning: family donors should not be used if healthy } \\
\text { carriers of the disease }\end{array}$ & Y & $\mathrm{N}$ \\
\hline Metachromatic leukodystrophy & & Y & $\mathrm{N}$ \\
\hline Immunodeficiencies & $\begin{array}{l}\text { We strongly suggest following ESID recommendations } \\
\text { for conditioning therapies https://esid.org }\end{array}$ & & \\
\hline Severe combined immunodeficiency & & Y & $\mathrm{N}$ \\
\hline $\begin{array}{c}\text { Severe combined immunodeficiency due to ADA } \\
\text { deficiency }\end{array}$ & Alternative: enzyme replacement and gene therapy & Y & $\mathrm{N}$ \\
\hline Wiskott Aldrich syndrome & & Y & $\mathrm{N}$ \\
\hline Familial hemophagocytic lymphohistiocytosis & $\mathrm{HCT}$ with active disease has worse results & Y & $\mathrm{N}$ \\
\hline Chediak-Higashi syndrome & & Y & $\mathrm{N}$ \\
\hline Griscelli syndrome - type II & & Y & $\mathrm{N}$ \\
\hline X-linked lymphoproliferative disease & & Y & $\mathrm{N}$ \\
\hline Chronic granulomatous disease & $\begin{array}{l}\text { HCT from mismatched unrelated donors or cord blood } \\
\text { have inferior results }\end{array}$ & Y & $\mathrm{N}$ \\
\hline HyperlgM Syndrome (CD40/CD40L) & & Y & $\mathrm{N}$ \\
\hline Leaky- Severe combined immunodeficiency & & Y & $\mathrm{N}$ \\
\hline Leukocyte adhesion deficiency & & Y & $\mathrm{N}$ \\
\hline Class II MHC deficiency & & Y & $\mathrm{N}$ \\
\hline $\begin{array}{l}\text { Purine nucleoside phosphorylase } \\
\text { (PNP) deficiency }\end{array}$ & & Y & $\mathrm{N}$ \\
\hline Complete gamma-interferon receptor deficiency & Attention to higher graft failure rate & Y & $\mathrm{N}$ \\
\hline Severe congenital neutropenia & $\begin{array}{l}\text { Patients refractory to GCSF, with a history of major } \\
\text { infections }\end{array}$ & Y & $\mathrm{N}$ \\
\hline $\begin{array}{l}\text { Early-onset inflammatory bowel disease (IL10, IL10-R, } \\
\text { XIAP) }\end{array}$ & & Y & $\mathrm{N}$ \\
\hline $\begin{array}{l}\text { Immunodysregulation polyendocrinopathy } \\
\text { enteropathy X-linked (IPEX) syndrome }\end{array}$ & & Y & $\mathrm{N}$ \\
\hline $\begin{array}{l}\text { Other immunoregulation defects (CTLA4, LRBA, STAT3 } \\
\text { GOF) }\end{array}$ & Some may have good response to immunobiologicals & Y & $\mathrm{N}$ \\
\hline Langerhans cell histiocytosis & $\begin{array}{l}\text { Multisystemic involvement beyond first remission or 1st } \\
\text { remission of refractory disease }\end{array}$ & Y & $\mathrm{N}$ \\
\hline
\end{tabular}


TABLE 2 - Indications for Hematopoietic Stem Cell Transplantation for Malignant Hematological Pediatric Diseases

\begin{tabular}{|c|c|c|c|}
\hline & & Allo & Auto \\
\hline \multicolumn{4}{|l|}{ Acute myeloid leukemia } \\
\hline 1 st remission & $\begin{array}{l}\text { Only if minimal residual disease }>0.1 \% \text { after second induction } \\
\text { or unfavorable genetics (karyotype or molecular findings) }\end{array}$ & Y & $\mathrm{N}$ \\
\hline $\begin{array}{l}\text { 2nd remission, persistent disease after } \\
\text { 2nd induction/ refractory disease }\end{array}$ & & Y & $\mathrm{N}$ \\
\hline \multicolumn{4}{|l|}{ Acute promyelocytic leukemia } \\
\hline 1 st remission & & $\mathrm{N}$ & $\mathrm{N}$ \\
\hline $\begin{array}{l}>\text { 2nd remission or persistently positive } \\
\text { PML-RARA }\end{array}$ & $\begin{array}{l}\text { If PML-RARA negative, autologous HCT. } \\
\text { If PML-RARA positive, allogeneic } \mathrm{HCT}\end{array}$ & Y & $\mathrm{Y}$ \\
\hline \multicolumn{4}{|l|}{ Acute lymphoblastic leukemia } \\
\hline \multicolumn{4}{|l|}{ 1st remission } \\
\hline $\begin{array}{l}\mathrm{Ph}+\text { Acute Lymphoblastic Leukemia (Bcr/ } \\
\text { Abl) }\end{array}$ & Only if there is not a good response to treatment & Y & $\mathrm{N}$ \\
\hline Hypodiploid & Only if there is not a good response to treatment & $\mathrm{Y}$ & $\mathrm{N}$ \\
\hline Age $<6$ months and KMT2A (MLL) positive & $\begin{array}{l}\text { Only if }>300,000 \text { leukocytes } / \mathrm{mm} 3 \text { or poor response to } \\
\text { corticosteroid }\end{array}$ & Y & $\mathrm{N}$ \\
\hline $\begin{array}{l}\text { Inductive failure (M2/M3 marrow) after } 4 \\
\text { weeks of treatment }\end{array}$ & Except if hyperdiploid and age $<6$ years & $\mathrm{Y}$ & $\mathrm{N}$ \\
\hline $\begin{array}{l}\text { Minimal Residual Disease (MRD) } \\
\geq 10-3(0.1 \%) \text { at the end of consolidation }\end{array}$ & & Y & $\mathrm{N}$ \\
\hline \multicolumn{4}{|l|}{ 2nd remission } \\
\hline T-lineage & Any relapse & Y & $\mathrm{N}$ \\
\hline \multicolumn{4}{|l|}{ B-lineage } \\
\hline \multirow[t]{2}{*}{ Isolated or combined medullary relapse } & Early: 1 st remission $<36$ months & $\mathrm{Y}$ & $\mathrm{N}$ \\
\hline & $\begin{array}{c}\text { Late: } 1 \text { st remission }>36 \text { months only if positive minimal } \\
\text { residual disease }\end{array}$ & Y & $\mathrm{N}$ \\
\hline \multirow[t]{2}{*}{ Isolated extramedullary relapse } & Early: 1st remission $<18$ months & Y & $\mathrm{N}$ \\
\hline & Late: 1 st remission $>18$ months & $\mathrm{N}$ & $\mathrm{N}$ \\
\hline 3rd remission (B or $\mathrm{T}$ lineages) & & Y & $\mathrm{N}$ \\
\hline Refractory disease & Absence of morphological remission & $\mathrm{N}$ & $\mathrm{N}$ \\
\hline \multicolumn{4}{|l|}{ Chronic myeloid leukemia } \\
\hline \multirow[t]{2}{*}{ 1st chronic phase } & $\begin{array}{l}\text { Only if therapeutic failure (lack of response or intolerance) is the } \\
\text { tyrosine kinase inhibitor }\end{array}$ & $\mathrm{Y}$ & $\mathrm{N}$ \\
\hline & Mutation T315I & $\mathrm{Y}$ & $\mathrm{N}$ \\
\hline Accelerated phase & & $\mathrm{Y}$ & $\mathrm{N}$ \\
\hline Blast crisis & In 2nd chronic phase & $\mathrm{Y}$ & $\mathrm{N}$ \\
\hline \multicolumn{4}{|l|}{ Myelodysplastic syndrome } \\
\hline Refractory cytopenia & $\begin{array}{l}\text { Only if unfavorable karyotype, transfusion dependence or } \\
\text { severe neutropenia }\end{array}$ & $\mathrm{Y}$ & $\mathrm{N}$ \\
\hline Advanced stages & & $\mathrm{Y}$ & $\mathrm{N}$ \\
\hline Any MDS secondary to chemotherapy & & $\mathrm{Y}$ & $\mathrm{N}$ \\
\hline Juvenile myelomonocytic leukemia & $\begin{array}{l}\text { Except Noonan syndrome or germline CBL with spontaneous } \\
\text { remission. }\end{array}$ & Y & $\mathrm{N}$ \\
\hline $\begin{array}{l}\text { Lymphomas } \\
\text { Burkitt, diffuse large B cell, anaplastic large } \\
\text { cell, Hodgkin lymphoma }\end{array}$ & $\begin{array}{l}\text { Only if poor response to treatment or } 2 \text { nd remission } \\
\text { If relapsed after autologous transplantation or failure to } \\
\text { mobilize autologous stem cells }\end{array}$ & $\mathrm{Y}$ & $\mathrm{N}$ \\
\hline
\end{tabular}


TABLE 3 - Indications for Autologous Hematopoietic Transplantation for Pediatric Solid Tumors

\begin{tabular}{|c|c|c|c|}
\hline Disease & $\begin{array}{l}\text { Stage of the disease with indication for autologous } \\
\text { transplantation }\end{array}$ & Auto & Allo \\
\hline \multirow[t]{2}{*}{ Neuroblastoma } & All patients with high-risk disease in 1st complete or partial remission & Y & $\mathrm{N}$ \\
\hline & $>2$ nd remission & $\mathrm{Y}$ & Y \\
\hline \multirow[t]{2}{*}{$\begin{array}{c}\text { Germ cell tumors: gonadal, } \\
\text { extra-gonadal and central } \\
\text { nervous system }\end{array}$} & In 1st remission only patients with unfavorable risk factors & Y & $\mathrm{N}$ \\
\hline & $>2$ nd complete or partial remission & $\mathrm{Y}$ & $\mathrm{N}$ \\
\hline Wilms tumor & $>2$ nd complete or partial remission & Y & $\mathrm{N}$ \\
\hline Clear Cell Sarcoma & $>1$ st complete or partial remission. Extremely aggressive tumor & Y & $\mathrm{N}$ \\
\hline \multirow[t]{2}{*}{ Ewing's sarcoma } & 1 st remission if unfavorable risk factors & $\mathrm{Y}$ & $\mathrm{N}$ \\
\hline & $>$ 2nd complete or partial remission & Y & $\mathrm{N}$ \\
\hline Alveolar soft part sarcoma & $>1$ st complete or partial remission & $\mathrm{Y}$ & $\mathrm{N}$ \\
\hline \multirow[t]{2}{*}{ Retinoblastoma } & $>1$ st remission of extra-ocular disease & Y & $\mathrm{N}$ \\
\hline & $>1$ st trilateral disease remission & Y & $\mathrm{N}$ \\
\hline Pinealoblastoma & $>1$ st complete or partial remission & Y & $\mathrm{N}$ \\
\hline Rhabdoid teratoid tumor & $\begin{array}{l}>1 \text { st complete or partial remission of central or extracranial nervous } \\
\text { system disease }\end{array}$ & Y & $\mathrm{N}$ \\
\hline \multirow[t]{2}{*}{ Medulloblastoma } & $\begin{array}{l}\text { 1st complete or partial remission in young children as an option for } \\
\text { radiotherapy, except for low-risk disease }\end{array}$ & $\mathrm{Y}$ & $\mathrm{N}$ \\
\hline & $>$ 2nd complete or partial remission & Y & $\mathrm{N}$ \\
\hline Choroid Plexus Carcinoma & $>$ 2nd complete or partial remission & Y & $\mathrm{N}$ \\
\hline
\end{tabular}


TABLE 4 - High-cost drugs that are fundamental to transplant and unavailable in the domestic market or for specific indications in bone marrow transplantation

\begin{tabular}{|c|c|}
\hline Medicine/ Procedure & Use \\
\hline Thiotepa & $\begin{array}{l}\text { Single chemotherapy that achieves optimal concentration in the } \\
\text { cerebrospinal fluid and brain parenchyma }\end{array}$ \\
\hline Treosulfan & Similar to busulfan, but significantly less toxic \\
\hline Defibrotide & Sinusoidal obstruction syndrome \\
\hline Eculizumab & $\begin{array}{l}\text { Single effective treatment for post-HCT thrombotic microangiopathy, extremely } \\
\text { serious complication }\end{array}$ \\
\hline Graft versus host disease & \\
\hline Mycophenolate mofetil & Prevention and treatment of graft-versus-host disease, IV presentation is unavailable \\
\hline Tacrolimus & Prevention and treatment of graft-versus-host disease \\
\hline Ruxolitinib & Treatment of refractory graft-versus-host disease \\
\hline Ibrutinib & Treatment of refractory graft-versus-host disease \\
\hline Extracorporeal photopheresis & Treatment of refractory graft-versus-host disease \\
\hline Antivirals & \\
\hline Cidofovir & Single antiviral with activity against poliomavirus and adenovirus \\
\hline Probenecid & Combination with cidofovir, increase bioavailability and decrease renal toxicity \\
\hline Foscarnet & Ganciclovir-resistant cytomegalovirus (CMV) infection \\
\hline Ribavirin (IV and inhaled) & $\begin{array}{l}\text { Single antiviral with spectrum against respiratory syncytial virus, unavailable in our } \\
\text { country }\end{array}$ \\
\hline Palivizumab & Specific immunoglobulin anti- respiratory syncytial respiratory virus \\
\hline Pentamidine & $\begin{array}{c}\text { Prevention and treatment of Pneumocystis jirovecii pneumonia in patients with G6PD } \\
\text { deficiency }\end{array}$ \\
\hline
\end{tabular}


SUPPLEMENTARY TABLE 1 - Participation of pediatricians in other groups:

\begin{tabular}{|c|c|}
\hline Pathology & Participants \\
\hline Acquired and Hereditary Bone Marrow Failure Syndromes & Carmem Bonfim, Luiz Guilherme Darrigo Jr \\
\hline Hemoglobinopathies & $\begin{array}{l}\text { Luiz Guilherme Darrigo Jr, Julia Lopes Garcia, Ana Karine Vieira, } \\
\text { Laila Rigolin }\end{array}$ \\
\hline Autoimmune diseases & Luiz Guilherme Darrigo Jr \\
\hline High-cost medications & Luiz Guilherme Darrigo Jr, Antonio Vaz de Macedo \\
\hline Acute lymphoblastic leukemia & Liane Daudt, Claudio Galvão \\
\hline Acute myeloid leukemia & Ana Luiza Melo Rodrigues \\
\hline Graft-versus-host disease & Rita Barbosa Tavares \\
\hline SOS/VOD & Gabriele Zamperlini, Natalia Borges \\
\hline
\end{tabular}


SUPPLEMENTARY TABLE 2 - Participation of the Pediatric Groups:

\begin{tabular}{|c|c|c|}
\hline \multicolumn{3}{|c|}{ Non-malignant diseases } \\
\hline Coordinator & Diagnosis & Participants \\
\hline \multirow{4}{*}{$\begin{array}{l}\text { Carmem Bonfim } \\
\text { Juliana Folloni }\end{array}$} & Immunodeficiencies & \\
\hline & $\begin{array}{l}\text { Severe combined } \\
\text { immunodeficiency }\end{array}$ & Samantha Nichele \\
\hline & Other & Samantha Nichele \\
\hline & Hmephphagocytic lympho & Gabriele Zamperlini, Samantha Nichele \\
\hline \multirow{4}{*}{$\begin{array}{l}\text { Carmem Bonfim } \\
\text { Juliana Folloni }\end{array}$} & INBORN ERRORS & \\
\hline & Osteopetrosis & Alessandra Gomes \\
\hline & Mucopolysaccharidoses & Alessandra Gomes \\
\hline & $\begin{array}{l}\text { Adrenoleukodystrophy and other } \\
\text { leukodystrophies }\end{array}$ & Alessandra Gomes \\
\hline \multicolumn{3}{|c|}{ Malignant diseases } \\
\hline Coordinator & Diagnosis & Participants \\
\hline Liane Daudt & ALL & $\begin{array}{l}\text { Adriana Seber, Antônio Vaz de Macedo, Claudio Galvão, } \\
\text { Cinthya Rocha, Renata Guimaraes, Luciana Domingues, } \\
\text { Maria Gabriela Matos, Maura Ikoma, Virginio Fernandes }\end{array}$ \\
\hline Ana Luiza Melo & AML & $\begin{array}{c}\text { Antonella Zanette, Victor Zecchin, GELMAI - Ana Maria } \\
\text { Marinho da Silva, Maria Lucia Lee, Raul Ribeiro }\end{array}$ \\
\hline Roseane Gouveia & CML & Antonio Vaz de Macedo, Luciana Domingues, Paola Soriano \\
\hline Neysimelia Villela & JMML & $\begin{array}{l}\text { Patricia Ikeuti } \\
\text { Simone Franco }\end{array}$ \\
\hline Neysimelia Villela & MDS, JMML, and other MPS & $\begin{array}{c}\text { Carla Zanchetta, Gustavo Zamperlini, Roseane Gouveia, } \\
\text { Simone Franco, Patricia Ikeuti, Carla Zanchetta, Pediatric SMD } \\
\text { Group }\end{array}$ \\
\hline Carla Nolasco & Lymphomas & $\begin{array}{l}\text { Cilmara Kuwahara, Gabriele Zamperlini, Mariana Michalowsky, } \\
\text { Valeria Ginani }\end{array}$ \\
\hline $\begin{array}{l}\text { Monica Cypriano } \\
\text { Victor Zecchin }\end{array}$ & Histiocytosis & Gustavo Zamperlini, Monica Cypriano \\
\hline
\end{tabular}

\begin{tabular}{|c|c|c|}
\hline \multicolumn{3}{|c|}{ Solid tumors } \\
\hline Coordinator & Diagnosis & Participants \\
\hline $\begin{array}{l}\text { Claudio Galvão - } \\
\text { SOBOPE }\end{array}$ & $\begin{array}{c}\text { Neuroblastoma, Ewing, } \\
\text { Soft tissue sarcoma, } \\
\text { Rhabdomyosarcoma, } \\
\text { Osteosarcoma, Hepatoblastoma, } \\
\text { Extra ocular retinoblastoma, } \\
\text { Germ cell tumors, Brain tumors, } \\
\text { GCT }\end{array}$ & $\begin{array}{l}\text { Carla Nolasco, Fernanda Lima, Gabriele Zamperlini, } \\
\text { Karoline Helena da Silva, Lauro Gregianin (guest), } \\
\text { Mariana Michalowski (guest), Natalia Borges, Patricia } \\
\text { Ikeuti, Paulo Klinger, } \\
\text { Simone Franco }\end{array}$ \\
\hline High cost medications & \multicolumn{2}{|c|}{ Antonio Vaz de Macedo, Luiz Guilherme Darrigo Jr } \\
\hline
\end{tabular}




\section{REFERENCES}

1.Seber A, Bonfim CMS, Daudt LE, Gouveia RV, Ginani VC, Mauad M et al. Indicações de transplante de células-tronco hematopoéticas em pediatria: consenso apresentado no I Encontro de Diretrizes Brasileiras em Transplante de Células-Tronco Hematopoéticas - Sociedade Brasileira de Transplante de Medula Óssea, Rio de Janeiro, 2009. [Indications for pediatric hematopoietic stem cell transplantation: consensus presented at the First Meeting on Brazilian Hematopoietic Stem Cell Transplantation Guidelines - Brazilian Society of Bone Marrow Transplantation, Rio de Janeiro, 2009], Rev. Bras. Hematol. Hemoter. v.32, n.3, p. 225-239, 2010.

2.II MEETING OF SBTMO OF BRAZILIAN GUIDELINES ON HEMATOPOIETIC STEM CELL TRANSPLANTATION (Consensus 2012). ISBN: 97885-88902-17-6 available www.sbtmo.org.br accessed October 5, 2020

3.https://www.jbmtct.com.br/seer/index.php/jbmtct accessed on October 5, 2020

4.https://site.abto.org.br/publicacao/ano-xxvnum-2-jan-jun-de-2019, and https://site.abto. org.br/publicacao/ano-xxvi-no-2

5.D'Souza A, Fretham C, Lee SJ, et al. Current Use of and Trends in Hematopoietic Cell Transplantation in the United States. Biol Blood Marrow Transplant. v.20, p. s1083-8791, 30225-1, 2020. 11:S1083-8791(20)30225-1, doi: 10.1016/j. bbmt.2020.04.013, PMID 32438042

6.Luznik L, Jalla S, Engstrom LW, lannone R, Fuchs EJ. Durable engraftment of major histocompatibility complex-incompatible cells after nonmyeloablative conditioning with fludarabine, low-dose total body irradiation, and posttrans- plantation cyclophosphamide. Blood. v.98, n.12, p. 3456-64, 1 dec. 2001. doi: 10.1182/blood. v98.12.3456. PMID: 11719388.

7.O'Donnell PV, Luznik L, Jones RJ, Vogelsang GB, Leffell MS, Phelps $M$, et al. Nonmyeloablative bone marrow transplantation from partially HLA-mismatched related donors using Posttransplantation cyclophosphamide. Biol Blood Marrow Transplant. v.8, n.7, p. 377-86, 2002.

8.Rashidi A, Hamadani M, Zhang MJ, et al. Outcomes of haploidentical vs matched sibling transplantation for acute myeloid leukemia in first complete remission. Blood Adv. v.3, n.12, p.1826-2836, 2019. doi: 10.1182/bloodadvances.2019000050. PMID: 31201170; PMCID: PMC6595262

9. D'Souza A, Fretham C, Lee SJ, et al. Current Use of and Trends in Hematopoietic Cell Transplantation in the United States. Biol Blood Marrow Transplant. 2020 v,26, n.8, p. 177,182, 2020. doi:10.1016/j.bbmt.2020.04.013. Epub 2020 May 11. PMID: 32438042; PMCID: PMC7404814.

10.Fuchs EJ, O'Donnell PV, Eapen M, Logan BR, Antin JH, Dawson $\mathrm{P}$, et al Double unrelated umbilical cord blood versus HLA-haploidentical bone marrow transplantation (BMT CTN 1101). Blood, blood. 2020 Aug 31:blood.2020007535. doi: 10.1182/blood.2020007535. Epub ahead of print. PMID:32870242.

11.Kanate AS, Majhail NS, Savani BN, Bredeson C, Champlin RE, Crawford S,et al Indications for Hematopoietic Cell Transplantation and Immune Effector Cell Therapy: Guidelines from the American Society for Transplantation and Cellular Therapy. Biol Blood Marrow Transplant. v.26, n.7, p.1247, 1256, jul.2020 